\title{
Willingness of the General Public to Receive the COVID-19 Vaccine During a Second-Level Alert - Beijing Municipality, China, May 2020
}

\author{
Rui Ma'; Luodan Suo ${ }^{1}$; Li Lu ${ }^{1, *}$; Xinghuo Pang ${ }^{1}$
}

\section{Summary}

What is already known on this topic?

Preclinical trials showed the effectiveness of domestic inactivated vaccine candidates for coronavirus disease 2019 (COVID-19). However, it is necessary to evaluate the willingness of the public to receive future domestic vaccines and to understand factors associated with willingness at the early stages of vaccine development.

What is added by this report?

Through May 25, 2020, $70.48 \%$ were willing to receive future domestic COVID-19 vaccines. Confidence in vaccines had the largest impact on public willingness, while age and presence of underlying chronic disease did not significantly increase public willingness.

What are the implications for public health practice?

It is necessary to increase awareness of COVID-19 vaccines among people with high risk of severe infection and to build public confidence in vaccines. Releasing accurate, timely, and reliable data to the public can help increase willingness to get vaccinated.

Preclinical animal studies showed that domestic inactivated vaccine candidates induced coronavirus disease 2019 (COVID-19) specific neutralizing antibodies, raising the possibility that mass vaccination with domestic vaccines might be used in the future to end the pandemic. This research conducted a survey among the public with a WeChat mini program (an application within WeChat) to determine intention to get vaccinated. Approximately $70.48 \%$ were willing to be vaccinated. Concerns about vaccine safety and effectiveness were the most important factors influencing willingness. Older age and presence of underlying chronic disease were not shown to significantly increase public willingness. Timely and accurate scientific data are greatly needed to build public confidence in vaccines, especially among people at high risk of severe COVID-19 infection. Immunization clinics may need increased resources to ensure high vaccination coverage.

On April 16, 2020, Beijing reported the city's first locally transmitted COVID-19 case. By April 30, 2020, Beijing reported no new cases for 14 consecutive days and lowered the COVID-19 emergency response from the highest level to the second highest level. As COVID-19 began spreading globally, Beijing faced an increasing risk of transmission of imported COVID-19 virus, also known as SARS-CoV-2. In May, 2020, domestic inactivated vaccines entered Phase II clinical trials in healthy adults 18 years of age and older (1). Preclinical animal studies had shown that inactivated vaccines induced COVID-19-specific neutralizing antibodies in animals and had a protective effect with no observed antibody-dependent enhancement of infection (ADE) (2). The research conducted a survey between May 12, 2020 and May 25, 2020 to determine willingness of the general public to get a future COVID-19 vaccine.

The study was an exploratory cross-sectional survey in 2 urban districts and 3 rural districts of Beijing. Respondents were classified into 5 age groups: 18-30, $31-40,41-50,51-60$, and $>61$ years old. We assumed an intention to get vaccinated $(p)$ to be $50 \%$, a maximum permissible error $(\delta)$ to be $10 \%$, and an allowable $\alpha$ error of $5 \%$. The estimated sample size for each age group was 385 according to the formula $n=\left(\frac{u_{\alpha}^{2} \times p \times(1-p)}{\delta^{2}}\right)$. The study surveyed at least 77 adults per age group in each district. We selected three townships with the largest population sizes. In each selected township, we selected the community with the largest population. Subjects were recruited by community committees. Two-dimensional barcodes were distributed to residential groups in WeChat, the mostly widely and frequently used mobile app for social communication in China (3). Respondents 
scanned the barcodes and completed the questionnaire on WeChat. The survey was brief to help ensure response quality and completeness - it took subjects only two minutes to answer all questions, decreasing the survey abandonment rate. Each mobile phone could only be used once to answer questions. The number of respondents was tallied daily. The survey ended when the number of subjects in each age group and each district reached their targets.

The questionnaires were designed to obtain information on respondent willingness to be vaccinated with a future domestic COVID-19 vaccine, the most trusted sources of information, preferred vaccination venue, and demographics. Logic skip patterns and data completeness checks were set in WeChat. Study procedures were approved by the Institutional Review Board and Human Research Ethic Committee of Beijing Center for Disease Prevention and Control. Informed consent was obtained at the beginning of the survey. Intention to receive a future vaccine was the primary outcome of the survey, scored as "No", "Uncertain", or "Yes". Descriptive statistics was used to summarize results. Multinomial logistic regression was used to identify factors associated with intention to receive a future vaccine. The main outcome of "Yes" (willing) was used as the referent. Statistical analyses were conducted with SPSS software (version 18.0, SPSS Inc, Chicago, IL, USA).

A total of 3,208 adults were surveyed. More than $30 \%$ of respondents were not sure that domestic COVID-19 vaccines were safe and effective. Among all respondents, $70.48 \%(2,261 / 3,208)$ were willing to get vaccinated, $23.66 \%(759 / 3,208)$ were uncertain, and $5.86 \%(188 / 3.208)$ were not willing to get vaccinated. Willingness varied by demographics, perception of COVID-19 disease, and vaccine characteristics. Among people aged $>60$ years, $74.41 \%$ were willing to get vaccination. Among people with underlying chronic disease, $73.02 \%$ were willing to get vaccinated. The 3 factors associated with the highest rate of willingness (above 80\%) were belief that vaccines were safe, belief that vaccines were effective, and whether they had received influenza vaccination during the most recent 3 years. Among people who thought vaccines were unsafe or ineffective, approximately $40 \%$ were unwilling to get vaccinated (Table 1).

TABLE 1. Demographic characteristics, perceptions of disease and domestic COVID-19 vaccines, and willingness to get a future vaccine in Beijing, China.

\begin{tabular}{|c|c|c|c|c|c|}
\hline \multirow[b]{2}{*}{ Variable } & \multirow[b]{2}{*}{ Number of interviewees (\%) } & \multicolumn{3}{|c|}{ Willingness to accept vaccination } & \multirow[b]{2}{*}{$P$ value } \\
\hline & & $\begin{array}{l}\text { "No", } \\
\text { n (\%) }\end{array}$ & $\begin{array}{c}\text { "Uncertain", } \\
\text { n (\%) }\end{array}$ & $\begin{array}{c}\text { "Yes", } \\
\text { n (\%) }\end{array}$ & \\
\hline \multicolumn{6}{|l|}{ Gender } \\
\hline Female & $1,950(60.79)$ & $111(5.70)$ & $500(25.64)$ & $1,339(68.67)$ & \multirow{2}{*}{0.004} \\
\hline Male & $1,258(39.21)$ & $77(6.12)$ & $259(20.59)$ & $922(73.29)$ & \\
\hline \multicolumn{6}{|l|}{ Age (years) } \\
\hline $18-30$ & $571(17.80)$ & $30(5.25)$ & $135(23.64)$ & $406(71.10)$ & \multirow{5}{*}{$<0.001$} \\
\hline $31-40$ & $1,050(32.73)$ & $56(5.33)$ & $284(27.05)$ & $710(67.62)$ & \\
\hline $41-50$ & $666(20.76)$ & $36(5.41)$ & $152(22.82)$ & $478(71.77)$ & \\
\hline $51-60$ & $456(14.21)$ & $34(7.46)$ & $101(22.15)$ & $321(70.39)$ & \\
\hline$>60$ & $465(14.50)$ & $32(6.88)$ & $87(18.71)$ & $346(74.41)$ & \\
\hline \multicolumn{6}{|l|}{ Highest education } \\
\hline Secondary school or lower & $474(14.78)$ & $25(5.27)$ & $94(19.83)$ & $355(74.89)$ & \multirow{3}{*}{$<0.001$} \\
\hline 3 -years of college & $1,301(40.55)$ & $69(5.30)$ & $278(21.37)$ & $954(73.33)$ & \\
\hline Undergraduate or higher & $1,433(44.67)$ & $94(6.56)$ & $387(27.01)$ & $952(66.43)$ & \\
\hline \multicolumn{6}{|l|}{ Living area } \\
\hline Urban & $1,821(56.76)$ & $97(5.33)$ & $491(26.96)$ & $1,233(67.71)$ & \multirow{2}{*}{$<0.001$} \\
\hline Suburban & $1,387(43.24)$ & $91(6.56)$ & $268(19.32)$ & $1,028(74.12)$ & \\
\hline \multicolumn{6}{|l|}{ Income } \\
\hline$<5,000 \mathrm{CNY}$ (700 USD) & $1,526(47.57)$ & $92(6.03)$ & $337(22.08)$ & $1,097(71.89)$ & \multirow{2}{*}{0.134} \\
\hline$\geq 5,000$ CNY (700 USD) & $1,682(52.43)$ & $96(5.71)$ & $422(25.09)$ & $1,164(69.20)$ & \\
\hline
\end{tabular}


TABLE 1. (Continued)

\begin{tabular}{|c|c|c|c|c|c|}
\hline \multirow[b]{2}{*}{ Variable } & \multirow[b]{2}{*}{ Number of interviewees (\%) } & \multicolumn{3}{|c|}{ Willingness to accept vaccination } & \multirow[b]{2}{*}{$P$ value* } \\
\hline & & $\begin{array}{l}\text { "No", } \\
\text { n (\%) }\end{array}$ & $\begin{array}{c}\text { "Uncertain", } \\
\text { n (\%) }\end{array}$ & $\begin{array}{c}\text { "Yes", } \\
\text { n (\%) }\end{array}$ & \\
\hline \multicolumn{6}{|l|}{ Underlying chronic disease } \\
\hline Yes & $430(13.40)$ & $37(8.60)$ & $79(18.37)$ & $314(73.02)$ & \multirow{2}{*}{0.002} \\
\hline No & $2,778(86.60)$ & $151(5.44)$ & $680(24.48)$ & $1,947(70.09)$ & \\
\hline \multicolumn{6}{|c|}{ Seasonal flu vaccination within 3 years } \\
\hline Yes & $488(15.21)$ & $32(8.44)$ & $41(10.82)$ & $306(80.74)$ & \multirow{2}{*}{$<0.001$} \\
\hline No & $2,720(84.79)$ & $156(5.51)$ & $718(25.38)$ & $1,955(69.11)$ & \\
\hline \multicolumn{6}{|c|}{ Perception of seriousness of COVID-19 disease } \\
\hline Very serious & $2,227(69.42)$ & $117(5.25)$ & $494(22.18)$ & $1,616(72.56)$ & \multirow{3}{*}{$<0.001$} \\
\hline Serious & $869(27.09)$ & $55(6.33)$ & $234(26.93)$ & $580(66.74)$ & \\
\hline Not serious & $112(3.49)$ & $16(14.29)$ & $31(27.68)$ & $65(58.04)$ & \\
\hline \multicolumn{6}{|c|}{ Perception of risk of contracting COVID-19 } \\
\hline Very likely & $276(8.60)$ & $18(6.52)$ & $46(16.67)$ & $212(76.81)$ & \multirow{3}{*}{0.020} \\
\hline Likely & $1,467(45.73)$ & $73(4.98)$ & $355(24.20)$ & $1,039(70.82)$ & \\
\hline Unlikely & $1,465(45.67)$ & $97(6.62)$ & $358(24.44)$ & $1,010(68.94)$ & \\
\hline \multicolumn{6}{|c|}{ If infected, my symptoms would be more severe than other people's } \\
\hline Yes & $417(13.00)$ & $21(5.04)$ & $68(16.31)$ & $328(78.66)$ & \multirow{3}{*}{$<0.001$} \\
\hline Uncertain & $1,847(57.57)$ & $74(4.01)$ & $484(26.20)$ & $1,289(69.79)$ & \\
\hline No & $944(29.43)$ & $93(9.85)$ & $207(21.93)$ & $644(68.22)$ & \\
\hline \multicolumn{6}{|c|}{ Perception of impact of COVID-19 pandemic on own life within the past 3 months } \\
\hline Very serious & $835(26.03)$ & $112(5.56)$ & $437(21.68)$ & $1,467(72.77)$ & \multirow{3}{*}{$<0.001$} \\
\hline Serious & $1,181(36.81)$ & $49(5.08)$ & $263(27.28)$ & $652(67.63)$ & \\
\hline Not serious & $1,192(37.16)$ & $27(11.84)$ & $59(25.88)$ & $142(62.28)$ & \\
\hline \multicolumn{6}{|c|}{ Perception of impact of COVID-19 pandemic on own life in the next 6 months } \\
\hline Very serious & $390(12.16)$ & $86(6.35)$ & $295(21.79)$ & $973(71.86)$ & \multirow{3}{*}{0.039} \\
\hline Serious & $964(30.05)$ & $69(4.72)$ & $357(24.42)$ & $1,036(70.86)$ & \\
\hline Not serious & $1,854(57.79)$ & $33(8.42)$ & $107(27.30)$ & $252(64.29)$ & \\
\hline \multicolumn{6}{|l|}{ Perception of vaccine safety } \\
\hline Safe & $2,147(66.93)$ & $85(3.96)$ & $307(14.30)$ & $1,755(81.74)$ & \multirow{3}{*}{$<0.001$} \\
\hline Uncertain & $1,028(32.04)$ & $87(8.46)$ & $444(43.19)$ & $497(48.35)$ & \\
\hline Unsafe & $33(1.03)$ & $16(48.48)$ & $8(24.24)$ & $9(27.27)$ & \\
\hline \multicolumn{6}{|c|}{ Perception of vaccine effectiveness } \\
\hline Effective & $2,189(68.24)$ & $87(3.97)$ & $314(14.34)$ & $1,788(81.68)$ & \multirow{3}{*}{$<0.001$} \\
\hline Uncertain & $1,000(31.17)$ & $93(9.30)$ & $440(44.00)$ & $467(46.70)$ & \\
\hline Ineffective & $19(0.59)$ & $8(42.11)$ & $5(26.32)$ & $6(31.58)$ & \\
\hline \multicolumn{6}{|c|}{ Perception of rebound of COVID-19 infection in China } \\
\hline Likely & $632(19.70)$ & $63(9.97)$ & $121(19.15)$ & $448(70.89)$ & \multirow{3}{*}{$<0.001$} \\
\hline Uncertain & $1,596(49.75)$ & $73(4.57)$ & $448(28.07)$ & $1,075(67.36)$ & \\
\hline Unlikely & $980(30.55)$ & $52(5.31)$ & $190(19.39)$ & $738(75.31)$ & \\
\hline Perception of continuity of & transmission & & & & \\
\hline Likely & $1,555(48.47)$ & $107(6.88)$ & $337(21.67)$ & $1,111(71.45)$ & \\
\hline Uncertain & $1,195(37.25)$ & $50(4.18)$ & $327(27.36)$ & $818(68.45)$ & $<0.001$ \\
\hline Unlikely & $458(14.28)$ & $31(6.77)$ & $95(20.74)$ & $332(72.49)$ & \\
\hline
\end{tabular}

*: $x^{2}$ test. 
Compared with the referent (willing) group, belief that vaccines were not safe was the most strongly associated factor for vaccine hesitancy and refusal, with adjusted odds ratio (OR) values of 2.86 and 13.33, respectively. People who had chronic diseases, who thought COVID-19 infection was not serious, who thought their symptoms would be less severe than others if infected, who thought their life had not been seriously affected during the previous three months, or who thought COVID-19 was likely to rebound in China, were more likely to refuse vaccines. Being uncertain of vaccine effectiveness was the second most associated factor for vaccine hesitancy, with an adjusted OR value of 2.68 (Table 2 ).

TABLE 2. Factors associated with intention to get a future domestic COVID-19 vaccine in Beijing, China.

\begin{tabular}{|c|c|c|c|c|}
\hline \multirow{2}{*}{ Variable } & \multicolumn{2}{|c|}{ Unwillingness* } & \multicolumn{2}{|c|}{ Uncertainty* } \\
\hline & Unadjusted OR (95\%Cl) & Adjusted OR (95\%Cl) & Unadjusted OR $(95 \% \mathrm{Cl})$ & Adjusted OR $(95 \% \mathrm{Cl})$ \\
\hline \multicolumn{5}{|l|}{ Gender } \\
\hline Female & 1 & 1 & 1 & 1 \\
\hline Male & $1.01(0.74-1.36)$ & $0.91(0.65-1.26)$ & $0.75(0.63-0.89)$ & $0.88(0.72-1.06)$ \\
\hline \multicolumn{5}{|l|}{ Age (years) } \\
\hline $18-30$ & 1 & $-^{\dagger}$ & 1 & $-^{\dagger}$ \\
\hline $31-40$ & $1.07(0.67-1.69)$ & $--^{\dagger}$ & $1.20(0.95-1.53)$ & $-\dagger$ \\
\hline $41-50$ & $1.02(0.62-1.68)$ & $-\dagger$ & $0.96(0.73-1.25)$ & $-{ }^{\dagger}$ \\
\hline $51-60$ & $1.43(0.86-2.39)$ & $--^{\dagger}$ & $0.95(0.70-1.27)$ & $-\dagger$ \\
\hline$>60$ & $1.25(0.75-2.10)$ & $--^{\dagger}$ & $1.20(0.95-1.53)$ & $-{ }^{\dagger}$ \\
\hline \multicolumn{5}{|l|}{ Highest education } \\
\hline Secondary school or lower & 1 & 1 & 1 & 1 \\
\hline 3-years of college & $1.03(0.64-1.65)$ & $1.54(0.92-2.59)$ & $1.10(0.85-1.43)$ & $1.06(0.79-1.43)$ \\
\hline Undergraduate or higher & $1.40(0.89-2.22)$ & $1.26(0.75-2.13)$ & $1.54(1.19-1.98)$ & $1.39(1.03-1.86)$ \\
\hline \multicolumn{5}{|l|}{ Living area } \\
\hline Urban & 1 & 1 & 1 & 1 \\
\hline Suburban & $1.13(0.84-1.52)$ & $1.28(0.92-1.79)$ & $0.66(0.55-0.78)$ & $0.79(0.65-0.96)$ \\
\hline \multicolumn{5}{|l|}{ Income } \\
\hline$<5,000$ CNY (700 USD) & 1 & 1 & 1 & 1 \\
\hline$\geq 5,000$ CNY (700 USD) & $0.98(0.73-1.32)$ & $--^{\dagger}$ & $1.18(1.00-1.39)$ & $--^{\dagger}$ \\
\hline \multicolumn{5}{|l|}{ Underlying chronic disease } \\
\hline Yes & 1 & 1 & 1 & 1 \\
\hline No & $0.66(0.45-0.96)$ & $0.50(0.32-0.78)$ & $1.39(1.07-1.80)$ & $1.17(0.86-1.58)$ \\
\hline \multicolumn{5}{|c|}{ Seasonal flu vaccination within 3 years } \\
\hline Yes & 1 & 1 & 1 & 1 \\
\hline No & $0.76(0.51-1.14)$ & $0.69(0.44-1.07)$ & $2.74(1.96-3.84)$ & $2.28(1.59-3.27)$ \\
\hline \multicolumn{5}{|c|}{ Perception of seriousness of COVID-19 disease } \\
\hline Very serious & 1 & 1 & 1 & 1 \\
\hline Serious & $1.31(0.94-1.83)$ & $1.25(0.88-1.79)$ & $1.32(1.10-1.58)$ & $1.33(1.08-1.63)$ \\
\hline Not serious & $3.40(1.91-6.06)$ & $2.27(1.18-4.37)$ & $1.56(1.01-2.42)$ & $1.39(0.86-2.27)$ \\
\hline \multicolumn{5}{|c|}{ Perception of risk in contracting COVID-19 } \\
\hline Very likely & 1 & 1 & 1 & 1 \\
\hline Likely & $0.83(0.48-1.42)$ & $0.86(0.48-1.56)$ & $1.58(1.12-2.21)$ & $1.17(0.80-1.70)$ \\
\hline Unlikely & $1.13(0.67-1.91)$ & $1.12(0.61-2.07)$ & $1.63(1.16-2.30)$ & $1.31(0.89-1.93)$ \\
\hline \multicolumn{5}{|c|}{ If infected, my symptoms would be more severe than other people's } \\
\hline Yes & 1 & 1 & 1 & 1 \\
\hline
\end{tabular}


TABLE 2. (Continued)

\begin{tabular}{|c|c|c|c|c|}
\hline \multirow{2}{*}{ Variable } & \multicolumn{2}{|c|}{ Unwillingness* $^{*}$} & \multicolumn{2}{|c|}{ Uncertainty* } \\
\hline & Unadjusted OR (95\% Cl) & Adjusted OR (95\% Cl) & Unadjusted OR (95\% Cl) & Adjusted OR (95\% Cl) \\
\hline Uncertain & $0.90(0.54-1.48)$ & $1.22(0.70-2.12)$ & $1.81(1.37-2.40)$ & $1.38(1.01-1.91)$ \\
\hline No & $2.26(1.38-3.69)$ & $3.05(1.71-5.45)$ & $1.55(1.14-2.10)$ & $1.23(0.86-1.76)$ \\
\hline \multicolumn{5}{|c|}{ Perception of impact of COVID-19 pandemic on own life within the past 3 months } \\
\hline Very serious & 1 & 1 & 1 & 1 \\
\hline Serious & $0.98(0.70-1.39)$ & $1.16(0.75-1.80)$ & $1.35(1.13-1.62)$ & $1.28(1.01-1.61)$ \\
\hline not serious & $2.49(1.58-3.92)$ & $2.54(1.35-4.78)$ & $1.40(1.01-1.92)$ & $1.17(0.77-1.78)$ \\
\hline \multicolumn{5}{|c|}{ Perception of impact of COVID-19 pandemic on own life in the next 6 months } \\
\hline Very serious & 1 & 1 & 1 & 1 \\
\hline Serious & $0.75(0.54-1.05)$ & $0.77(0.51-1.16)$ & $1.14(0.95-1.36)$ & $1.07(0.85-1.34)$ \\
\hline Not serious & $1.48(0.97-2.27)$ & $1.06(0.58-1.95)$ & $1.40(1.08-1.82)$ & $1.39(0.97-1.97)$ \\
\hline \multicolumn{5}{|c|}{ Perception of vaccine safety } \\
\hline Safe & 1 & 1 & 1 & 1 \\
\hline Uncertain & $3.61(2.64-4.95)$ & $2.35(1.44-3.83)$ & $5.11(4.28-6.09)$ & $2.50(1.91-3.27)$ \\
\hline Unsafe & $36.71(15.77-85.47)$ & $13.33(4.83-36.80)$ & $5.08(1.95-13.27)$ & $2.86(1.02-7.96)$ \\
\hline \multicolumn{5}{|c|}{ Perception of vaccine effectiveness } \\
\hline Effective & 1 & 1 & 1 & 1 \\
\hline Uncertain & $4.09(3.00-5.58)$ & $2.52(1.55-4.10)$ & $5.37(4.50-6.40)$ & $2.68(2.05-3.50)$ \\
\hline Ineffective & $27.40(9.30-80.73)$ & $3.52(0.94-13.17)$ & $4.75(1.44-15.64)$ & $3.28(0.92-11.77)$ \\
\hline \multicolumn{5}{|c|}{ Perception of rebound of COVID-19 infection in China } \\
\hline Likely & 1 & 1 & 1 & 1 \\
\hline Uncertain & $0.48(0.34-0.69)$ & $0.57(0.37-0.89)$ & $1.54(1.23-1.94)$ & $1.23(0.93-1.62)$ \\
\hline Unlikely & $0.50(0.34-0.74)$ & $0.56(0.35-0.92)$ & $0.95(0.74-1.23)$ & $1.08(0.79-1.48)$ \\
\hline \multicolumn{5}{|c|}{ Perception of continuity of global COVID-19 transmission } \\
\hline Likely & 1 & 1 & 1 & 1 \\
\hline Uncertain & $0.64(0.45-0.90)$ & $0.63(0.41-0.97)$ & $1.32(1.10-1.57)$ & $0.96(0.77-1.20)$ \\
\hline Unlikely & $0.97(0.64-1.47)$ & $1.16(0.70-1.92)$ & $0.94(0.73-1.22)$ & $1.12(0.83-1.52)$ \\
\hline
\end{tabular}

${ }^{*}:$ Being willing to get vaccine was selected as reference category in multinomial logistic regression.

${ }^{\dagger}$ : Variables that were not statistically significant in univariate analyses were excluded from the multinomial logistic regression model.

Respondents' most popular sources of information about COVID-19 vaccines were social media $(86.94 \%$, $2,789 / 3,208)$, medical doctors $(78.68 \%, 2,524 / 3,208)$, and professional papers $(34.57 \%, 1,109 / 3,208)$. Among the 2,261 respondents who were willing to get vaccinated, $58.29 \%(1,318 / 2,261)$ preferred getting vaccinated in immunization clinics.

\section{DISCUSSION}

This study found that $70 \%$ of the general public were willing to be vaccinated with a COVID-19 vaccine, a rate close to the $74 \%$ willingness found in a study conducted in France at about the same time as our survey (4). A study in Wuhan city showed that the basic reproduction number $\left(\mathrm{R}_{0}\right)$ of COVID-19 was $2.24-3.58$ (5) in the early phase of the epidemic, indicating that $55.36 \%-72.07 \%$ of the population needs to be immune to the virus to prevent sustained transmission. Based on that result, the future COVID-19 vaccination rate should be at least $70 \%$ in Beijing, assuming that COVID-19 vaccines are $70 \%-80 \%$ effective in preventing disease. In our study, about $70 \%$ were willing to get COVID-19 vaccines, which is close to that target. We also found that $20 \%$ of the general public was uncertain whether they would get a COVID-19 vaccine. Among people who believed vaccines were safe and effective, $81 \%$ were willing to get vaccinated. Therefore, achieving the goal of no sustained spread of COVID-19 seems not far off in Beijing through use of a mass vaccination program. In 
January 2021, Beijing started a COVID-19 vaccination campaign targeting people aged 18-59 years old, and shortly thereafter extended the age range to 60 years and above. At the time of publication of this article, coverage of the 1 st dose of COVID-19 vaccine has exceeded the $70 \%$ coverage level that was predicted in our study, as more than $80 \%$ of people over 18 years of age have already received at least one dose of COVID-19 vaccine in the ongoing campaign (6).

Among interviewees, more than $30 \%$ were not confident in COVID-19 vaccines - a finding that may be due to lack of scientific data at the time the survey was conducted. "Vaccine hesitancy" ( 7 ) could be another reason. Our multivariable analyses showed confidence in vaccine safety had the highest impact on public willingness. Although belief that the vaccine was ineffective did not significantly increase the unwillingness rate, the $\mathrm{OR}$ value was 27.40 , and statistically significant in univariate analyses. Being uncertain of vaccine effectiveness was also significantly associated with vaccine hesitancy (Table 2). Therefore, building confidence in domestic vaccines should be a priority. It was noteworthy that age had no significant impact on willingness. It is therefore important to increase awareness of vaccines among people $\geq 60$ years of age, who were more likely to have severe COVID19 (8). People who thought COVID-19 was serious or who thought their symptoms would be more severe than others if infected, were more willing to get vaccinated. This finding implies that social mobilization, especially among people with high risk of severe COVID-19, could increase vaccine acceptance. If vaccines are shown to be effective against severe infection, willingness to get vaccines may significantly increase. Presence of underlying chronic diseases was associated with an increased possibility to refuse vaccination. That could be due to concerns that vaccination may exacerbate the disease. It is difficult to explain our finding that people who believed COVID19 might rebound in China were more likely to refuse vaccination. Further study on this point is needed. The finding does suggest that social mobilization is necessary among people believing that COVID-19 will rebound in China.

More than $70 \%$ of the general public received vaccine information from social media or medical doctors. Media reports therefore should be objective and fair. Increased willingness of medical doctors to get vaccinated could be another key factor. Around one third of the public received information from professional literature. Hence, accurate, timely, and reliable data about vaccines should be released to the general public through social media and medical doctors.

Over half of respondents preferred getting vaccinated in clinics that provide routine immunization for children aged $0-14$ years. Increased resources and personnel for immunization clinics would be necessary to avoid decreases in coverage of routine vaccines during mass COVID-19 vaccination.

Social mobilization has played an important role in achieving high coverage in Beijing's vaccination campaign. Consistent with findings in our study, building public confidence in vaccines through publicity of the COVID-19 vaccine clinical trials that showed the vaccines to be safe and effective, making vaccination convenient by establishing temporary vaccination clinics, and using social media to increase awareness of the importance of immunization have been instrumental in building high coverage levels.

Our study has some limitations. First, our result could not be generalized to the entire adult population. Second, our results can only be applied to when Beijing set its public health emergency response to the second level and data of Phase I clinical trials of domestic COVID-19 vaccines were available. Continued monitoring will be important. Third, selfreported data may have introduced information bias.

Our study showed a high level of willingness of the general public to be vaccinated with domestic COVID-19 vaccines. Building public confidence in vaccines through social media and medical doctors is needed. It is also necessary to increase awareness of vaccines among people with high risk of severe COVID-19 infections. Increased personnel and resources for routine immunization clinics should be considered to prepare for mass vaccination efforts.

Acknowledgements: Shijingshan District Center for Disease Control and Prevention, Fangshan District Center for Disease Control and Prevention, Mentougou District Center for Disease Control and Prevention, Tongzhou District Center for Disease Control and Prevention, and Pinggu District Center for Disease Control and Prevention.

doi: $10.46234 / \mathrm{ccdcw} 2021.118$

\# Corresponding author: Li Lu, lulibj@sina.com.

\footnotetext{
${ }^{1}$ Division of Expanded Program of Immunization (EPI), Beijing Center for Disease Control and Prevention/Beijing Center for Preventive Medicine Research, Beijing, China.
}

Submitted: March 03, 2021; Accepted: May 18, 2021 


\section{REFERENCES}

1. World Health Organization. Draft landscape and tracker of COVID-19 candidate vaccines. https://www.who.int/who-documents-detail/draftlandscape-of-covid-19-candidate-vaccines. [2020-6-5].

2. Gao Q, Bao LL, Mao HY, Wang L, Xu KW, Yang MN, et al. Development of an inactivated vaccine candidate for SARS-CoV-2. Science 2020;369(6499):77-81. http://dx.doi.org/10.1126/science. abc1932.

3. Zhang XT, Wen D, Liang J, Lei JB. How the public uses social media wechat to obtain health information in China: a survey study. BMC Med Inform Decis Mak 2017;17(Suppl 2):66. http://dx.doi.org/10.1186/ s12911-017-0470-0.

4. The COCONEL Group. A future vaccination campaign against COVID-19 at risk of vaccine hesitancy and politicisation. Lancet Infect Dis 2020;20(7):769 - 70. http://dx.doi.org/10.1016/S1473-3099(20)
30426-6.

5. Zhao S, Lin QY, Ran JJ, Musa SS, Yang GP, et al. Preliminary estimation of the basic reproduction number of novel coronavirus (2019$\mathrm{nCoV}$ ) in China, from 2019 to 2020: a data-driven analysis in the early phase of the outbreak. Int J Infect Dis 2020;92:214-7. http://dx. doi.org/10.1016/j.ijid.2020.01.050.

6. Beijing Municipal Health Commission, Over $80 \%$ of adults 18 years and older have received at least one dose of the COVID-19 vaccine in Beijing. Available online: http://www.beijing.gov.cn/ywdt/gzdt/202105/ t20210519_2392459.html. [2021-5-19]. (In Chinese).

7. Yang RH, Penders B, Horstman K. Addressing vaccine hesitancy in China: a scoping review of Chinese scholarship. Vaccines 2019;8(1):2. http://dx.doi.org/10.3390/vaccines8010002.

8. Guan WJ, Ni ZY, Hu Y, Liang WH, Ou CQ, He JX, et al. Clinical characteristics of coronavirus disease 2019 in China. N Engl J Med 2020;382(18):1708 - 20. http://dx.doi.org/10.1007/s11596-020-2172-6. 